\title{
A satellite magnetometer observation of dusk-to-dawn current in the midnight magnetosphere at low-latitude $\mathrm{Pi} 2$ onset
}

\author{
O. Saka ${ }^{1}$, H. Akaki ${ }^{2}$, and D. N. Baker ${ }^{3}$ \\ ${ }^{1}$ Department of Physics, Kurume National College of Technology, Kurume 830-8555, Japan \\ ${ }^{2}$ Department of Earth and Planetary Science, Kyushu University, Fukuoka 812-8581, Japan \\ ${ }^{3}$ Laboratory for Atmospheric and Space Physics, University of Colorado, Boulder, CO 80309, U.S.A.
}

(Received June 13, 2002; Revised August 25, 2002; Accepted September 11, 2002)

\begin{abstract}
A multiple Pi2 event was observed in the midnight sector at the low-latitude ground magnetometer station. Meanwhile, particle fluxes in the midnight sector were measured during the multiple Pi2 event by the AMPTE/CCE spacecrafts in the $L$ range from 4.8 to 5.8 and by geosynchronous satellite, S/C1984-129, at the dawn sector. We present an examination of the magnetic field changes and the associated particle behavior during the multiple Pi2 event. We argue that the temporal intensification of the dusk-to-dawn currents near the geosynchronous altitudes may trigger the Pi2 pulsations. The plasmas injected in the inner magnetosphere are possible candidate for driving the dusk-to-dawn currents.
\end{abstract}

Key words: Low-latitude Pi2, current system, midnight magnetosphere, substorm onset, particle injection.

\section{Introduction}

The onset of magnetospheric substorms is accompanied by many kinds of disturbances in the ionosphere and the magnetosphere (Baker et al., 1996). Auroral break-up, particle injections, and $\mathrm{Pi} 2$ magnetic pulsations are some of the observable manifestations at the substorm expansion onset, and numerous investigations about these manifestations have been carried out for the last few decades (Akasofu, 1964; Reeves et al., 1990; Yeoman et al., 1994; Saka et al., 1996a). Recently, bursty bulk flow (BBF) phenomena in the magnetotail have been considered to play an important role in causing substorm current system developed in the near-Earth tail region (Shiokawa et al., 1997). A high correlation of the occurrence of the BBF with Pi2 onset was shown by Hsu and McPherron (1996) on the basis of ISEE $1 / 2$ satellites data. They concluded that Pi2's may be generated by BBF's. The field line change at the Pi2 onset has been examined statistically by use of the magnetometer data at the geosynchronous orbit (Saka et al., 1998). They showed that the appearance of hot plasmas in the inner magnetosphere is another manifestation at the Pi2 onset. There appear to be a variety of possible sources of the hot plasmas in the magnetosphere, from injection boundary (McIlwain, 1974), local instability (Friedel et al., 1996) or transport from the near-Earth tail by compressional waves (Moore et al., 1981) or by transient electric fields (Birn et al., 1996).

Examination of the particle and field changes that occur at Pi2 onset in the inner magnetosphere $(L \sim 4.8-5.8)$ is carried out here by use of magnetometer and particle data from the AMPTE/CCE spacecraft. We argue that the tem-

Copy right $(\mathrm{C}$ The Society of Geomagnetism and Earth, Planetary and Space Sciences (SGEPSS); The Seismological Society of Japan; The Volcanological Society of Japan; The Geodetic Society of Japan; The Japanese Society for Planetary Sciences. poral intensification of the dusk-to-dawn currents, driven by the particle injection, may trigger the $\mathrm{Pi} 2$ pulsations.

\section{Observations}

A multiple Pi2 onset composed of eight Pi2 wave-packets was observed at the low-latitude ground magnetometer station, Kuju $\left(25.9^{\circ} \mathrm{N}, 202.4^{\circ}\right.$ in geomagnetic coordinates; $L=$ 1.24) for the period 1450-1550 UT, on 31 August 1986 (top panel in Fig. 1). Meanwhile, electrons in the energy channels ranging $30-140 \mathrm{keV}$ and protons in $63-710 \mathrm{keV}$ range (see lower panels in Fig. 1) have been observed by geosynchronous satellite (S/C1984-129) in the dawn sector and by the AMPTE/CCE spacecraft in the L range 4.98-5.78 at the midnight sector. There appeared flux oscillations in all energy channels of S/C1984-129 for the periods from 1445 UT to $1545 \mathrm{UT}$. These seem to be a possible instrumental noise. Nevertheless, we could look at the increase of the electron flux at 1511 UT in $95-140 \mathrm{keV}$ channel, 1514 UT in 65-95 $\mathrm{keV}$ channel, $1519 \mathrm{UT}$ in $45-65 \mathrm{keV}$ channel, and at 1528 UT in $30-45 \mathrm{keV}$ channel as marked by the arrows in the figure. Let us suppose that energetic electrons entrained in the midnight sector at $1500 \mathrm{UT}$, when the Pi2 marked A commenced. If we assume an energy dispersion of the eastward drift velocity of these electrons (1.4-2.1 LT/10 min for 30$45 \mathrm{keV}, 3.8-5.7 \mathrm{LT} / 10 \mathrm{~min}$ for 95-140 keV) (e.g., Saka et al., 1996b), the first rise of the electron flux as marked by arrows could be accounted for by the drift time of the electrons entrained at 1500 UT. The electron flux increased thereafter because fresh electrons that were supplied at the consecutive Pi2 onset drifted and overlapped at the satellite sector. While in the inner $\mathrm{L}$ range, the proton injection was not detected on board the AMPTE/CCE spacecraft.

The dynamic power spectra of the $H$ component (along the dipole axis) calculated by the Fast-Fourier Transform 


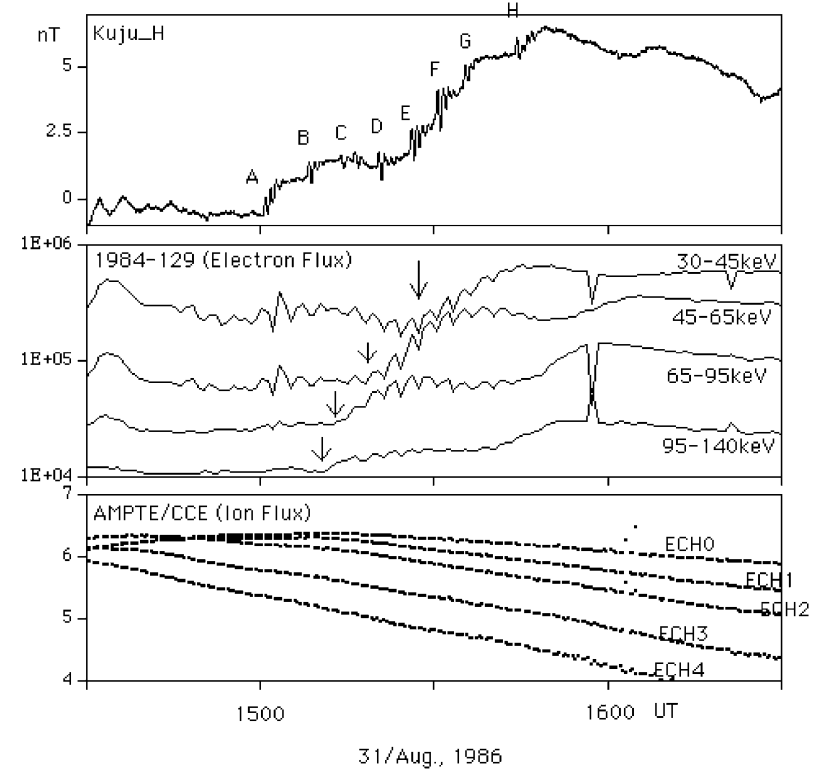

Fig. 1. Top panel: The multiple $\mathrm{Pi} 2$ event (labeled A through $\mathrm{H}$ ) as recorded by ground station, Kuju, in midnight sector $(\mathrm{LT}=\mathrm{UT}+9)$ for the time interval 1100-1700 UT of 31 August, 1986. Second panel: A differential energetic electron flux $\left(\mathrm{cm}^{-2} \mathrm{sec}^{-1} \mathrm{sr}^{-1}\right)$ averaged over one-minute interval (30-45 keV, 45-65 keV, 65-95 keV, 95-140 keV) observed at geosynchronous altitudes by S/C1984-129. The satellite was at 0438 LT sector at $1500 \mathrm{UT}$. The arrows in the panel indicate $\mathrm{Pi}$-associated injection onset in four energy channels, respectively. The oscillations in the plot are possible instrumental noise. Third panel: Ion flux variations (only a multiple number is shown) for the AMPTE/CCE energy channels (averaged over four spin periods), ECH0; 63-85 keV, ECH1; 63-85 keV, ECH2; 125-210 keV, ECH3; 210-380 keV, ECH4 $380-710 \mathrm{keV}$.

(FFT) technique (calculated every 13.2 min interval $(6.2 \mathrm{~s} \times$ 128 points), sliding by 1 min interval) are shown in the top panel of the Fig. 2 for the interval from 1430 UT to 1700 UT covering the multiple Pi2 event (1500-1540 UT). It is clear that the wave activity was seen to occur in the $15 \mathrm{mHz}$ band (shown by the horizontal arrow) which is in the same frequency band as the multiple Pi2 event in the ground magnetometer data. Therefore, we expect a simultaneous occurrence of the wave activity between the ground and space.

Let us look at an original wave form of the satellite data, $H, V, D$ components in the dipole coordinates in the lower panel of Fig. 2 for the selected interval 1455-1555 UT corresponding to the multiple $\mathrm{Pi} 2$ event; $H$ is along the dipole axis, $V$ is radial outward, and $D$ is azimuthally eastward. The magnitudes of the $H$ and $V$ components decreased smoothly in the satellite's outbound orbit; from $L=4.98$, $-8.3^{\circ}$ MLAT, $21.5 \mathrm{MLT}$ at $1500 \mathrm{UT}$ to $L=5.78,-8.3^{\circ}$ MLAT, 22.1 MLT at 1535 UT. The fluctuations of the order of $1-2 \mathrm{nT}$ in the Pi2 band are seen to be superimposed on the smooth change of the background level.

We examine such an association in detail by a close examination of the wave form at two locations. The results are presented in Fig. 3, wherein the satellite data were high-passfiltered to remove the background trend with a frequency cutoff at $1000 \mathrm{sec}$. In the figure, the wave form in the time interval covering the Pi 2 events labeled as A, B, E, and F (see Fig. 1) is examined. For the rest of the events, the Pi 2 wave form is obscured because it is overwhelmed with the large
$\mathrm{mHz} \quad$ CCE_H $1986 / 8 / 31$

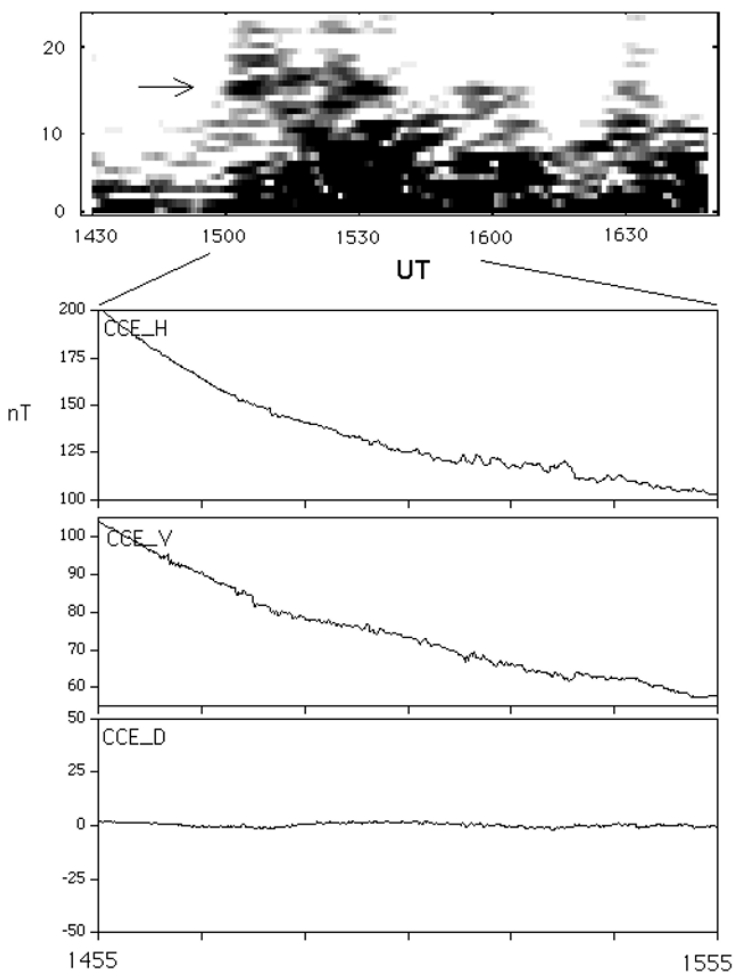

Fig. 2. Top: Dynamic power spectra for the $H$ component of the magnetic field data from AMPTE/CCE spacecraft ranging 1430-1700 UT. Bottom: The wave form of the $H, V, D$ components of AMPTE/CCE magnetic field data for the time period corresponding the multiple $\mathrm{Pi} 2$ event of 31 August, 1986 (1455-1555 UT). $H, V, D$ components are in the dipole coordinates; $H$ is along dipole axis, positive northward, $V$ is radially outward, $D$ is azimuthally eastward).

background fluctuations. Each event contains $H$ and $V$ components together with $B$ and Theta components, where $B$ is field magnitude, Theta is the field line inclination measured positive upward from the equatorial plane. The variations were plotted in 10 min intervals; 1455-1505 UT for A (first panel), 1505-1515 UT for B (second panel), and 1525-1535 UT for E, F (third panel). The initial peak of the amplitude as observed in the $H$ and $B$ components is marked by the solid line to emphasize the correlation with respect to the ground Pi 2 onset recorded in the $H$ component. The initial changes at the spacecraft $L$ indicate an increase of the $H$ and a decrease of the $V$ components, which resulted in an increase of the field magnitude $B$ and the field inclination Theta. The initial peak of the $B$ component at the satellite location as marked by the vertical line leads that of the ground $H$ component by approximately $20-28 \mathrm{sec}$.

Those initial field changes as observed by AMPTE/CCE spacecraft (field magnitude $B$ was increased, inclination was increased, $H$ was increased, and $V$ was decreased) is consistent with the field changes produced by the dusk-to-dawn current at the tailward of the satellite position (XGSM = -4.1 , YGSM $=2.9, \mathrm{ZGSM}=0.1$ in GSM coordinates) as illustrated in Fig. 4.

If the signals were propagating radially across the field lines down on the low-latitude station, the time-lag in the range of 20-28 sec may indicate a velocity of the order of 


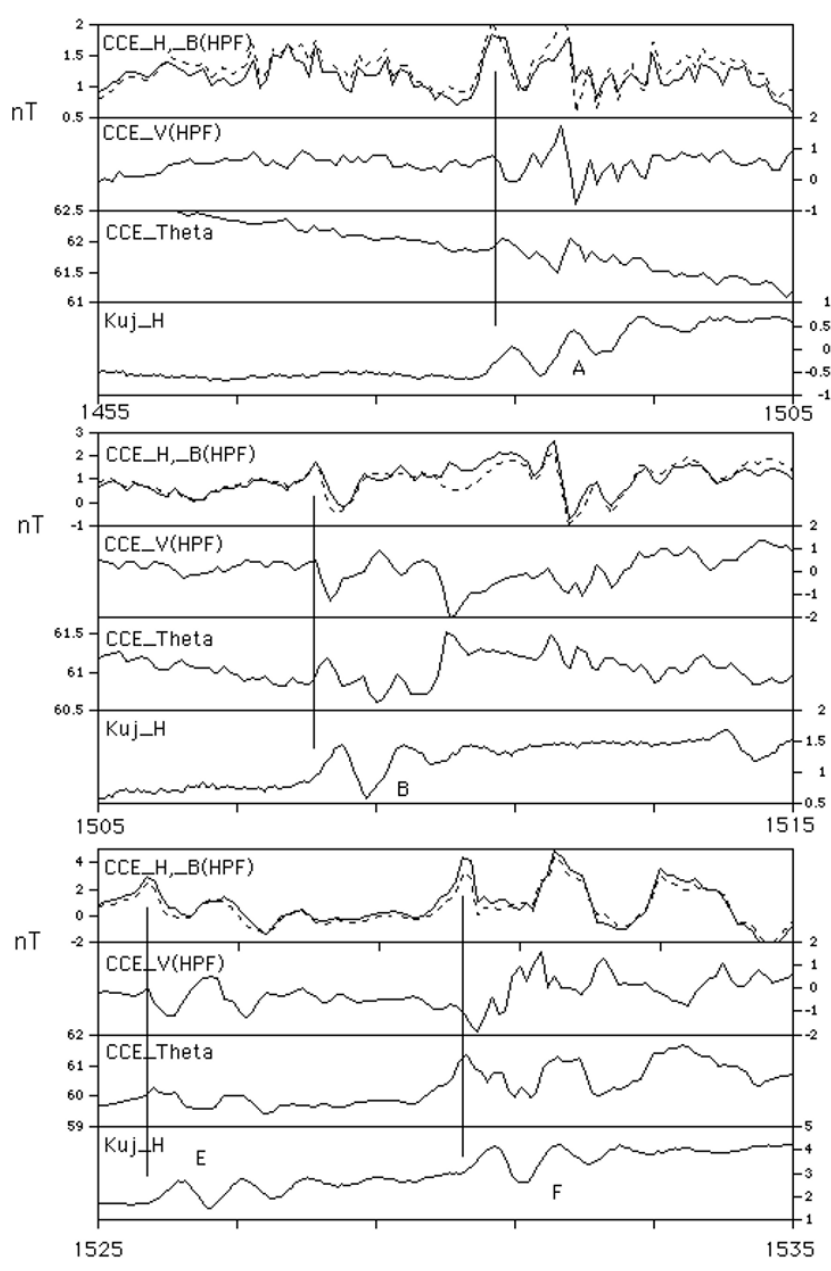

Fig. 3. Ground-satellite correlation of the Pi2 wave form. The high-pass-filtered data with cutoff at $1000 \mathrm{sec}$ were presented for AMPTE/CCE data for the event A, B, E, and F with enlarged time scale (10 $\mathrm{min})$. For each event; $H$ is positive northward along the dipole axis (solid line), while the dashed lines indicate the field magnitudes $B$ (upper panel); $V$ is radial outward (second panel); Theta is field inclination in degrees measured positive from the equatorial plane (third panel); the $H$ component of ground magnetometer station at Kuju (fourth panel). The vertical bars indicate an initial peak of the $H$ and $B$ component of the satellite data. $H, V$, and $B$ are plotted in nT.

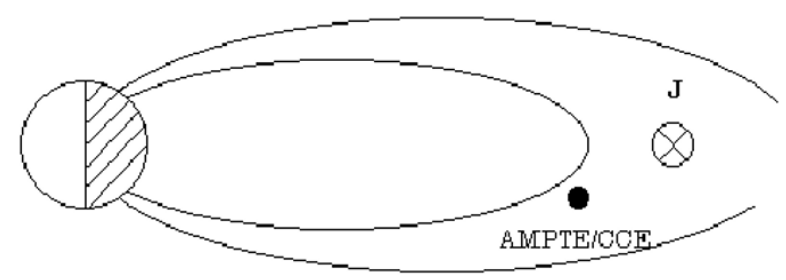

Fig. 4. Schematic illustration of meridional cross-section of midnight sector showing the location of the AMPTE/CCE spacecraft and of the possible dusk-to-dawn currents.

$\sim 1000 \mathrm{~km} / \mathrm{s}$. They would produce cavity/wave guide mode signatures on the surface of the earth (Allan et al., 1996).

\section{Discussions}

A statistical investigation of the magnetic field variations in the geosynchronous altitudes suggests that the dusk-todawn currents often appear in the vicinity or even earthward of the geosynchronous altitudes at Pi2 onset (Saka et al., 1998). We argue that the dusk-to-dawn currents are primary force arising in the inner magnetosphere to balance the injected plasmas supplied from the plasma sheet. Therefore, those dusk-to-dawn currents are supposed to be co-located at the earthward boundary of the injected plasmas. These dusk-to-dawn currents are responsible for the global cavity mode (e.g., Allan et al., 1996) or global poloidal mode (Fujita et al., 2000, 2002). For that reason, the mechanisms discussed here confined to the low-latitude Pi2s, though $\mathrm{Pi} 2 \mathrm{~s}$ distributed widely from the high latitude to the equator. For the present case, the plasmas have not been arrived at the inner satellite orbit $(L<5.8)$. Those dusk-to-dawn currents are likely to flow at a radial distance tailward of the AMPTE/CCE spacecraft as illustrated in Fig. 4. If the consecutive injection takes place in the midnight magnetosphere, the dusk-to-dawn currents could be intensified on all such occasions. The multiple Pi2 onset as reported in this study is attributed to such an injection pattern. The energetic electrons entrained the inner magnetosphere at the midnight sector execute the eastward drift. The particle detector on board the satellite remote from the midnight sector, such as S/C1984-129 at 0438 LT sector, records an obscured onset signature of the consecutive injection, as spatial profile of each injection overlap while they drifted eastward. If the geosynchronous satellite was in the midnight sector, however the particle detector on board the satellite often records the clear onset of the consecutive particle injection.

Acknowledgments. We are grateful to K. Takahashi (JHU/APL) for providing the AMPTE/CCE proton data. The CCE energetic ion data were provided by the AMPTE Science Data Center of JHU/APL. R. W. McEntire is the principle investigator of the particle experiment. The AMPTE/CCE magnetometer data are provided by T. A. Potemra (JHU/APL). The LANL energetic particle data were provided through G. D. Reeves.

\section{References}

Akasofu, S.-I., The development of auroral substorm, Planet. Space Sci., 12, 273, 1964

Allan, W., F. W. Menk, B. J. Fraser, Y. Li, and S. P. White, Are low-latitude Pi 2 pulsations cavity/waveguide modes?, Geophys. Res. Lett., 23, 765, 1996.

Baker, D. N., et al., Neutral line model of substorms: Past results and present view, J. Geophys. Res., 101, 12975, 1996.

Birn, J., et al., MHD simulations of magnetotail dynamics, J. Geophys. Res., 101, 12939, 1996.

Friedel, R. H., et al., Substorm onsets observed by CRRES: Determination of energetic particle source regions, J. Geophys. Res., 101, 13137, 1996.

Fujita, S., M. Itonaga, and H. Nakata. Relation between the Pi2 pulsations and the localized impulsive current associated with the current disruption in the magnetosphere, Earth Planets Space, 52, 267-281, 2000.

Fujita, S., H. Nakata, M. Itonaga, A. Yoshikawa, and T. Mizuta. A numerical simulation of the Pi2 pulsations associated with the substorm current wedge, J. Geophys. Res., 107, SMP2-1-2-15, 2002.

Hsu, T. S. and R. L. McPherron, Occurrence frequency of substorm field and plasma signatures observed near-earth by ISEE-1/2, in Proc. ICS-3, edited by E. J. Rolfe and B. Kaldeic, 1996.

McIlwain, C. E., Substorm injection boundaries, in Magnetospheric Physics, edited by B. M. McCormac, p. 143, D. Reidel, Hingham, Mass., 1974.

Moore, T. E., et al., Propagating substorm injection fronts, J. Geophys. Res., 86, 6713, 1981.

Reeves, G. D., T. A. Fritz, T. E. Cayton, and R. D. Belian, Multi-satellite measurements of the substorm injection region, Geophys. Res. Lett., 17, 2015, 1990.

Saka, O., H. Akaki, O. Watanabe, and D. N. Baker, Ground-satellite correla- 
tion of low-latitude Pi2 pulsations: A quasi-periodic field line oscillation in the magnetosphere, J. Geophys. Res., 101, 15433, 1996a.

Saka, O., O. Watanabe, and D. N. Baker, A possible driving source for transient field line oscillations in the postmidnight sector at geosynchronous altitudes, J. Geophys. Res., 101, 24719, 1996b.

Saka, O., et al., An initial response of magnetic fields at geosynchronous orbit to Pi 2 onset as observed from the dip-equator, Ann. Geophysicae, 16, 542, 1998.
Shiokawa, K., W. Baumjohann, and G. Haerendel, Braking of high-speed flows in the near-Earth tail, Geophys. Res. Lett., 24, 1179, 1997.

Yeoman, T. K., et al., A comparison of midlatitude Pi 2 pulsations and geostationary orbit particle injections as substorm indicators, J. Geophys. Res., 99, 4085, 1994.

O. Saka (e-mail: saka@kurume-nct.ac.jp), H. Akaki, and D. N. Baker 